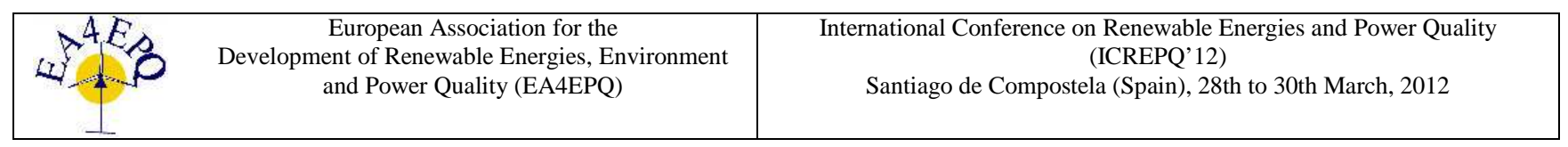

\title{
Dimming Compatibility of 60W Replacement LED Lamps with Legacy Dimming Infrastructure
}

\author{
A. Boura, M. Husak \\ Department of Microelectronics, Faculty of Electrical Engineering \\ Czech Technical University in Prague \\ Technicka 2, CZ - 16627 Prague 6, Czech Republic \\ Phone/Fax number: +420 224352237 / +420 224310 792, e-mail: bouraa@ fel.cvut.cz, husak@ fel.cvut.cz
}

\begin{abstract}
The paper summarizes basic problems of dimming compatibility of modern LED lamps with the legacy dimming infrastructure. In 2016 the legacy incandescent bulbs are going to be forbidden on the European market and thus it must be replaced by modern and energy efficient solid state lighting. The problems with dimming compatibility appeared. Standards phase-cutting dimmers are widely used in the households and consumers expect the modern lighting to have the same properties as the incandescent bulbs. The legacy dimmers are designed for some minimal load (usually $40-60 \mathrm{~W}$ ) while the modern LED lamps replacements have only about $12 \mathrm{~W}$ keeping the $60 \mathrm{~W}$ incandescent bulb luminous flux $(800 \mathrm{~lm})$. Several different problems must be solved in order to keep the dimming ability, avoid the light flickering and keep the dimmer in the safe operation region.

There are two basic dimmer types. The leading edge dimmers that are usually based on triac switch and trailing edge dimmers that use MOSFET switches and electronic control. Both types have its specific demands and need different approach for ensuring the dimming compatibility. New LED driver must be developed in order to be compatible with the legacy dimmers.
\end{abstract}

\section{Key words}

Incandescent $60 \mathrm{~W}$ replacement, Dimming compatibility, Solid State Lighting, Leading edge, Trailing edge

\section{Introduction}

Presented work was made within the project CSSL (Consumerizing Solid State Lighting [1]) which is aimed on development of a new affordable LED lighting to be equivalent to the $60 \mathrm{~W}$ incandescent bulbs. Regulations in Europe already ban the $100 \mathrm{~W}$ incandescent clear glass in 2009 and $60 \mathrm{~W}$ incandescent lamps in September 2011. All other incandescent lamps will be ban till 2016. There are basically two possible equivalent light sources that can be used instead of the incandescent ones. The Compact fluorescent lamp (CFL) and Light emitting diodes (LED). The CFL are already widely spread and relatively cheep but the light quality is not very comfortable and the manufacturing process is encumbered by the problematic and dangerous materials. Other possibility is to use the LED light sources which have excellent light quality, it is energy efficient but it is still very expensive. Typical price for incandescent bulb is $\$ 0.3$, for CFL $\$ 3.5$ and for LED lamp (Cool White) \$25.0 [1]. More over the SSL is still not fully compatible with the legacy household infrastructure. The CSSL project it targeting to deal with these problems.

This paper focuses on compatibility of the LED driver with the phase cutting dimmers. As it was already mentioned, the legacy dimmers are determined for minimal load about $40 \mathrm{~W}$, which is 3 times more then the modern efficient light sources. Other problem is with the loading impedance. The incandescent bulbs behave as a resistor while the LED driver has more likely capacitive character. Special approach must be used to keep the modern light sources compatible with the legacy dimmers.

There are four basic meanings of the word compatibility. The compatibility can be related to the following tasks that will be discussed in next chapters:
1) Consumers' expectations
2) LED driver performance
3) Dimmer performance
4) Powering infrastructure disturbances

Fulfil all the compatibility demands is a challenging task which has no satisfying solution yet. Main objective of this paper is to summarize the problems which can help in the new LED driver design.

\section{Consumers' expectations}

The consumers' expectations seem to be very simple. The SSL replacement must be cheep, the performance and the form factor must by same as the incandescent E27 screw base bulb, it must be compatible with all the dimmers, must have good quality of the light, it must be 
energy saving and applicable together with other types of the bulbs and lamps.

\section{A. Form factor}

Demand for similar form factor as the E27 Edison bulb brinks problems with the drivers' dimension limits. There is also problem with the thermal sink construction.

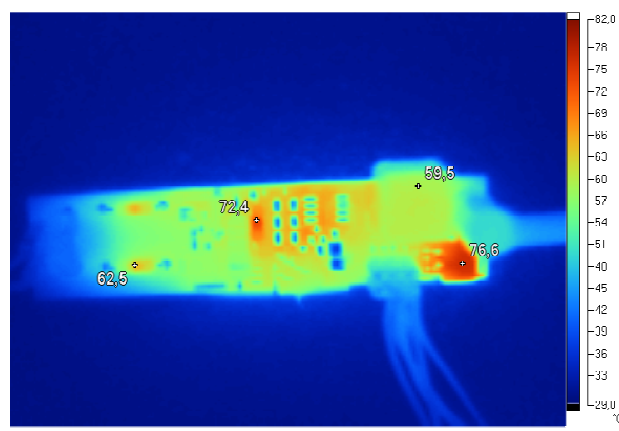

Fig. 1. Thermo camera image of the LED driver with hot spots

Figure 1 presents a thermo camera measurement of the LED driver which is partly compatible with the triac based dimmers. The dimming compatibility is fulfilled using the bleeding resistors that are loading the dimmer by current pulses [4, 5]. Hot spots on the drivers' PCB must be treated properly by adapting the heat sink [3]. Special attention must be paid on the electrical safety and isolation. All these factors are limiting the driver's dimensions.

\section{B. Dimming curve}

Dimming curve of the SSL must be similar to the incandescent bulbs or it can be fitted to the human perception (nonlinear curve). For the LED lamps the current to light transfer is linear but the human perception is nonlinear. The dimming curve should be preset electronically. Typical dependence of the incandescent bulb light dependence during the dimming is presented on the figure 2 .

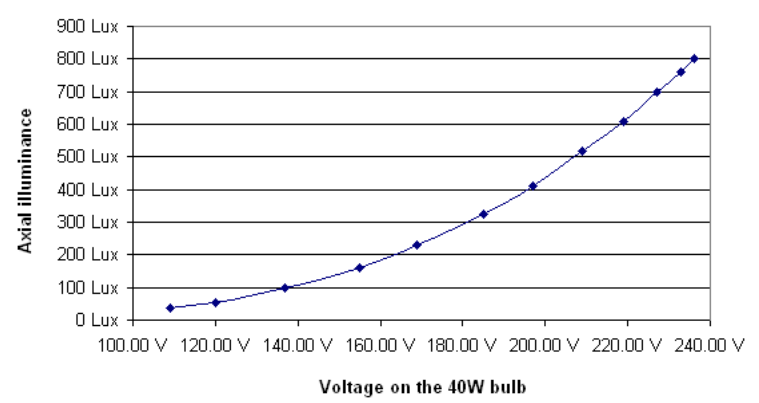

Fig. 2. Measurement of the $40 \mathrm{~W}$ incandescent bulb illuminance during dimming

Specific LED lamp can also implement the colour change during dimming (shift toward the red wavelengths), while the price of such LED lamp is increasing because standard white LEDs have constant colour.

Problematic part with setting the dimming characteristics is that each dimmer have different performance. Figure 3 presents dependence of the voltage during dimming for selected dimmers that are widely used in European households. The voltage on the $60 \mathrm{~W}$ bulb is related to the position of the dimmers' regulator. The maximal value is always the same, while the minimal voltage can vary a lot. More then twenty dimmers were measured. Typical minimal voltage was around $50 \mathrm{~V}$ but some of the dimmers have minimal value $10 \mathrm{~V}$ or $150 \mathrm{~V}$. General LED drivers thus can not have the same dimming performance for all the dimmers.

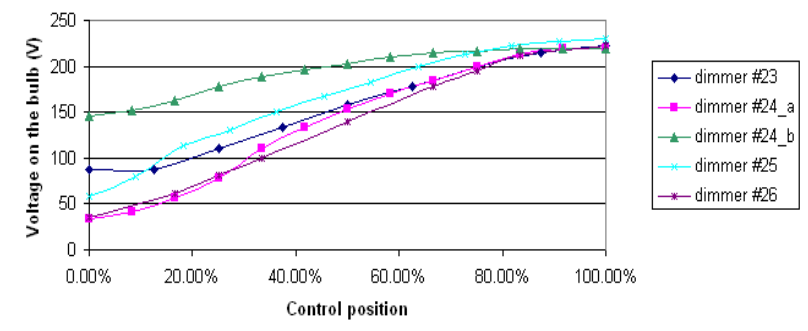

Fig. 3. Regulation range for incandescent $60 \mathrm{~W}$ bulb and different dimmers

The minimal intensity of the light must be some kind of compromise based on the average performance of the dimmers.

\section{Quality of the light}

The most important parameter of the light is the stable intensity. There must not be any visible flickering or light intensity change. Problem can appear when several different bulbs are used simultaneously. Then the color and intensity difference can be annoying, especially if products of different manufacturers are used simultaneously in the same area.

Lifetime of the LED lamp replacements is expected to be more then 25000 hours (about 3 years) [1]. Good color and light intensity standardization during the manufacturing process thus must be ensured at least for several years.

\section{Energy efficient performance}

There are two basic motivations for using the dimmers. One is the optimal light level setting and the second is the energy saving purpose. The consumer is expecting lower energy consumption when the dimmer is active. This can be problem, because the LED drivers' circuits, that are ensuring the dimming compatibility, are usually power demanding. The total power thus can be nearly the same as the nominal power consumption and the power is usually deflected from the LEDs to some kind of the bleeders during dimming. When the intelligent bleeder switching is used, the total power consumption can be partly reduced, but still the overall power consumption decrease during dimming is lower compared to the decrease when the incandescent bulb is used.

\section{LED driver side}

The LED driver is the key device for the SSL. It must be able to recognize the switching angle of the dimmer and 
also keep the dimmer in correct operation state. The LED light intensity can by set using the variable current or using the pulse width modulation.

There are two basic dimmer types - the leading edge dimmers and the trailing edge dimmers. The trailing edge dimmers with the capacitive loads can brink problems. When the sine wave on the mains is cut, the voltage is kept on the capacitive load and the LED driver can have problems with recognition of the switching angle. Figures 4 and 5 show this effect on the $60 \mathrm{~W}$ incandescent bulb and on the modern CSSL - 7 W, $2700 \mathrm{~K}$, Philips 7E27A60 lamp. Curves number 1 represent the voltage on the load that is controlled by the dimmer. Curves number 2 are the currents through the load and the curves number 3 represent the voltage of the mains before the dimmer.

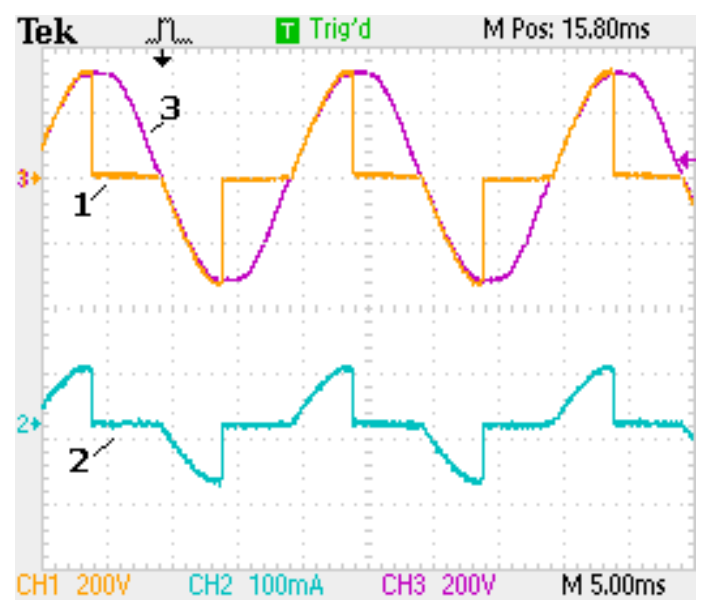

Fig. 4. Voltages and currents on the $60 \mathrm{~W}$ incandescent bulb during dimming using the trailing edge dimmer

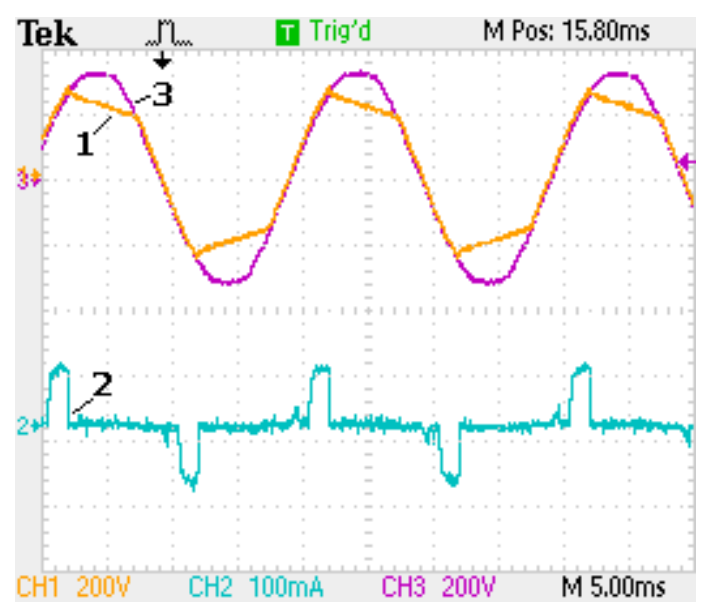

Fig. 5. Voltages and currents on the LED driver during dimming using the trailing edge dimmer

The capacitive character of the driver is changing the voltage curve shape. The switching angle thus must be periodically measured on the resistive bleeder or some different measurement must be implemented. According the measurements, the dimming curve (Fig. 2) for incandescent lamp is equal for leading and trailing edge dimmers. For dimmable LED lamp it is usually different.

Important part of the LED driver performance is also detection of the OFF state. Some wall switches can be equipped by the glow lamp which is slowly charging the input capacitor of the driver and the LED lamp slightly lights or the light bursts can occur.

\section{Dimmer side}

The dimmer must be kept in correct performance region. The dimmers are designed for some minimal load. If the load is too week the performance of the dimmer is going to be crippled and it can be also dangerous for the dimmer. The triac based dimmers are very sensitive on this load decrease because of the filtering inductance. Figure 6 presents a typical scheme of the triac-based dimmer.

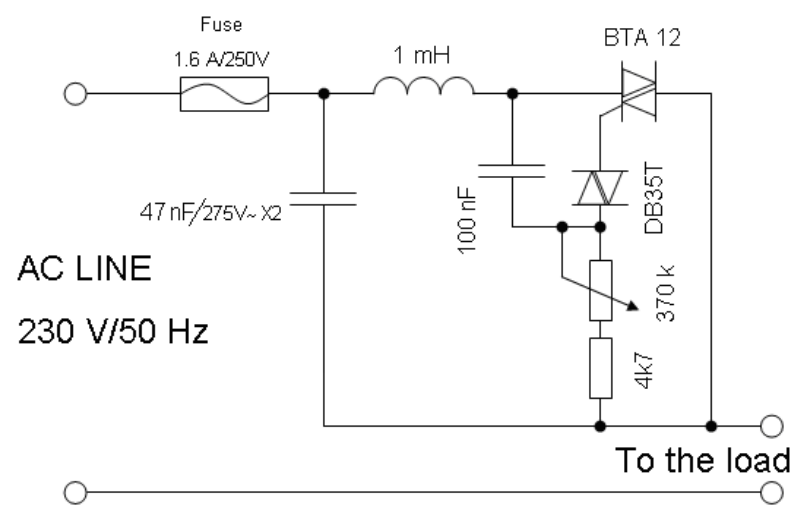

Fig. 6. Schematic of typical triac-based dimmer

When the loading current decreases below some specific value, the triac is closed. If the voltage on the input capacitor is high enough, an unstable situation can occur. The filtering inductance and the input capacitor can form a resonance tank circuit which periodically switches on the triac. The worst situation that can happen is presented on the figure 7 , where curve 1 represents the voltage on the load and curve number 2 is the current. The tiac is slightly asymmetrical device and the triac holding current can be different for each polarity. Figure 7 presents a situation when the load is going to be critical. For positive part of the period it is stable while for the negative part the holding current is too small and the periodical switching is presented. This unstable performance is causing flickering of the LED driver and it can be also dangerous for the dimmer (overheating, voltage peaks and current harmonics)

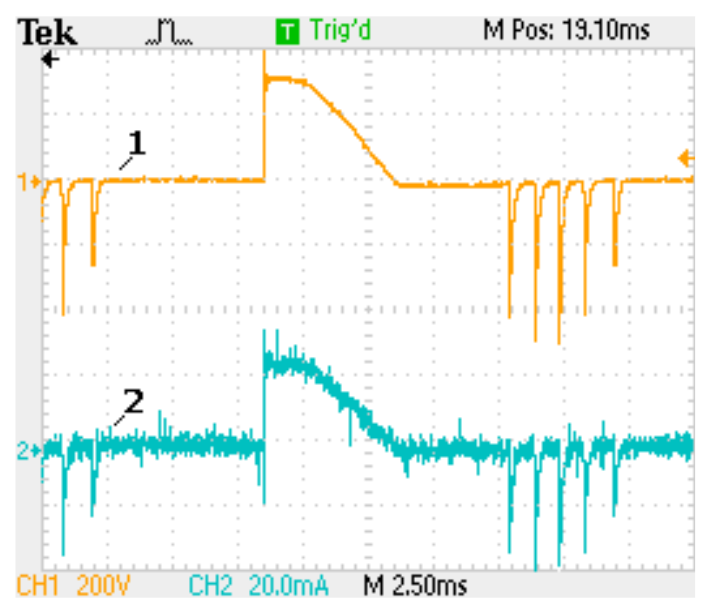

Fig. 7. Voltages and currents for week load on the triac-based dimmer 
In order to keep the triac-based dimmer in the correct performance area, some extra circuits must be designed. Typical way of doing that is intelligent switching of the bleeding resistors $[2,4,5]$ but it is increasing the power consumption. In order to keep the overall efficiency high, the current bleeders must be switched in moments, where the voltage is low and then the power is not increasing much.

Some modern dimmers contain an electronics which requires some minimal power. The electronics serves for switching ON and OFF or for providing a remote control. Powering of the electronics inside these dimmers used to be provided via the filament of the incandescent bulb. If the LED driver is connected to this kind of dimmer, some extra circuits must be implemented to guarantee the minimal current through the LED lamp when it is switched off. The typical off state current is about $5 \mathrm{~mA}$.

\section{Powering infrastructure}

Current peaks and harmonic distortion are typical products of the electronic drivers. These disturbances can spread in the powering infrastructure and cause problems. It is evident especially when several SSL bulbs are used simultaneously and no other resistive load is connected. The LED driver must be able to keep the power factor as high as possible.

The current peaks are most significant when the leading edge dimmers are used on driving angle $90^{\circ}$. Input capacitor of the LED driver is periodically charged by the full amplitude of the input voltage and it is producing the current peaks which can increase several hundreds of percents the nominal current consumption. Example of real measurement is presented on the figure 8 .

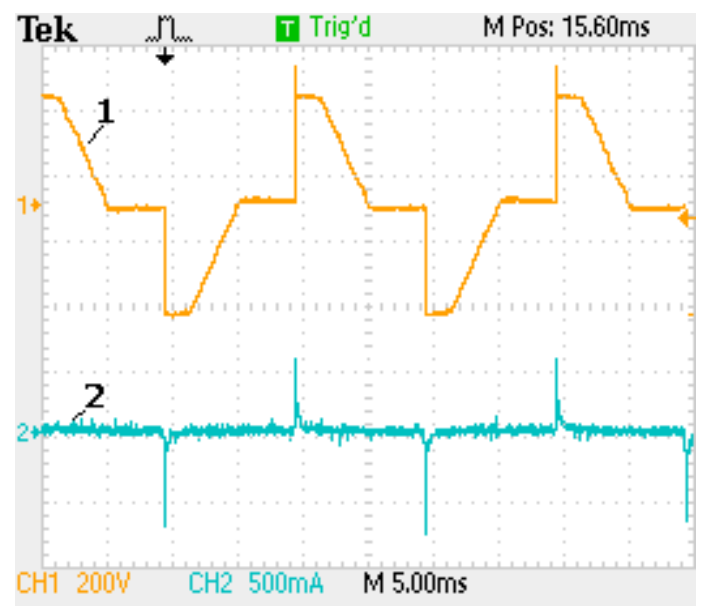

Fig. 8. Voltage (curve 1) and current (curve 2) of the $7 \mathrm{~W}$ CSSL lamp with dimmer Busch 2200 and driving angle 90

The bottom curve 2 is representing the LED lamp input current. Depending on the filtering inductance in the dimmer the current peaks can exceed several amperes.

There are two possible solution of this problem. It can be optimized the input capacitor of the driver, while it is limited by the LED driver consumption. Other possibility is to increase the input filtering inductance. This solution is expensive and there can be problem with the physical dimensions (it must fit into the E27 bulb form).

\section{Conclusion}

Paper presents basic problems with implementation of the modern solid state lighting instead of the incandescent lamps. The LED driver must fulfill different requirements which are hard to reach simultaneously. There exist several particular solutions but the final solution is still not satisfactory. Besides the mentioned leading and trailing edge dimmers there exists also universal type of the dimmers that are able to recognize the load character and according to the load it behaves as the leading edge or the trailing edge dimmer. These dimmers were also tested with new kinds of the LED drivers, that are using the strong and the week bleeders for dimming compatibility $[4,5,6]$. In some case the results were very bed. The intelligent dimmer was confused by the impulse character of the load and the behavior of such dimmers was unpredictable. It was flickering, blinking and in some cases it did not start to light.

Presented problems with the implementation of the SSL dimming compatibility can be generally described as the technical problems and as the problems with standardizations. Technical problems have particular solutions that must be improved to ensure the dimming compatibility with most of the dimmers on the market. The definitions and standardization must be performed in order to have universal replacement for the $60 \mathrm{~W}$ incandescent bulbs and ensure similar properties of the light and dimming performance across the manufacturers.

\section{Acknowledgement}

The work was supported by CSSL consortium which is partly funded by the European Commission and the participating Public Authorities in the ENIAC (European Nanoelectronics Initiative Advisory Council) Joint Undertaking.

\section{References}

[1] CSSL project web page: http://www.consumerizingssl.eu/

[2] Young, J.: "Configuring the NCL30000 for TRIAC Dimming", APPLICATION NOTE AND8448, December 2009, http://www.onsemi.com/

[3] Jakovenko, J. et. Al..: "SSL2102Thermal simulation and validation of 8W LED lamp", 2011 12th Int. Conf. on Thermal, Mechanical and Multi-Physics Simulation and Experiments in Microelectronics and Microsystems, EuroSimE 2011, Article number 5765818, ISBN: 978145770107-8

[4] NXP Semiconductors N.V.: "SSL2102, Mains LED driver IC for dimmable LED lighting", SSL2102_1 product data sheet, June 2009, http://www.nxp.com

[5] NXP Semiconductors N.V.: "AN10754 SSL2101 and SSL2102 dimmable mains LED driver", AN10754_3 Application note, October 2009, http://www.nxp.com

[6] National Electrical Manufacturers Association: "Solid State Lighting for Incandescent Replacement - Best Practices for Dimming", A NEMA Lighting Systems Division Document LSD 49-2010, 2010 